\title{
Effects of Emergency Department Expansion on Emergency Department Patient Flow
}

\author{
Bryn E. Mumma, MD, MAS, James Y. McCue, [none], Chin-Shang Li, PhD, and James F. \\ Holmes, MD, MPH \\ Department of Emergency Medicine (BEM, JFH), School of Medicine (JYM), and Department of \\ Public Health Sciences Division of Biostatistics, (CL) University of California Davis, Sacramento, \\ CA
}

\begin{abstract}
Objectives-Emergency department (ED) crowding is an increasing problem associated with adverse patient outcomes. ED expansion is one method advocated to reduce ED crowding. The objective of this analysis was to determine the effect of ED expansion on measures of ED crowding.
\end{abstract}

Methods-This was a retrospective study using administrative data from two 11-month periods before and after the expansion of an ED from 33 to 53 adult beds in an academic medical center. ED volume, staffing, and hospital admission and occupancy data were obtained from either the electronic health record or administrative records. The primary outcome was the rate of patients who left without being treated (LWBT), and the secondary outcome was total ED boarding time for admitted patients. A multivariable robust linear regression model was used to determine whether ED expansion was associated with the outcome measures.

Results-The mean daily adult volume was 128 (SD \pm 14 ) patients before expansion, and 145 ( $\mathrm{SD} \pm 17$ ) patients after. The percentage of patients who LWBT was unchanged: $9.0 \%$ before expansion, vs $8.3 \%$ after expansion (difference $0.6 \%, 95 \% \mathrm{CI}=-0.16 \%$ to $1.4 \%$ ). Total $\mathrm{ED}$ boarding time increased from 160 to 180 hours/day (difference 20 hours, $95 \% \mathrm{CI}=8$ to 32 hours). After adjusting for daily ED volume, low-acuity area volume, daily wait time, daily boarding hours, and nurse staffing, the decrease in patients who LWBT was not independently associated with ED expansion $(\mathrm{p}=0.053)$. After adjusting for ED admissions, ED intensive care unit admissions, elective surgical admissions, hospital occupancy rate, ICU occupancy rate, and number of operational ICU beds, the increase in ED boarding hours was independently associated with the ED expansion $(\mathrm{p}=0.005)$.

Conclusions-An increase in ED bed capacity was associated with no significant change in the percentage of patients who LWBT, but had an unintended consequence of an increase in ED boarding hours. ED expansion alone does not appear to be an adequate solution to ED crowding.

Corresponding Author: Bryn E. Mumma, MD, MAS, UC Davis Department of Emergency Medicine, 4150 V Street, PSSB \#2100, Sacramento, CA 95817, mummabe@gmail.com, Phone: 916-734-5010; Fax: 916-734-7950.

Presentations: Society for Academic Emergency Medicine Western Regional Meeting, Long Beach, CA, March 2013; and Society for Academic Emergency Medicine Annual Meeting, Atlanta, GA, May 2013

Conflicts of Interest: The authors have no relevant financial information or potential conflicts of interest to disclose. 


\section{INTRODUCTION}

Emergency department (ED) crowding is an increasing problem. ${ }^{1}$ The American College of Emergency Physicians defines crowding as occurring when "the identified need for emergency services exceeds available resources for patient care in the ED, hospital or both." 2 This phenomenon is fueled by rapidly growing numbers of ED visits combined with declining numbers of ED facilities. ${ }^{3}$ ED crowding is associated with adverse effects on patient outcomes, including delays in thrombolytics for acute myocardial infarction, 4,5 antibiotics for pneumonia, ${ }^{6,7}$ pain relief for numerous conditions, ${ }^{8-11}$ and treatment for acute asthma. ${ }^{12}$ It is also associated with higher rates of in-hospital adverse cardiac events, ${ }^{13}$ and mortality. ${ }^{14-16}$

Conceptual models of ED crowding divide critical determinants of ED crowding into input, throughput, and output. ${ }^{17} \mathrm{ED}$ input is driven by the demand for ED care, and includes emergency care, unscheduled acute care, and safety net care. ED throughput encompasses the triage, evaluation, and diagnostic steps that influence patient length of stay (LOS) in the ED. ED output focuses on the timely disposition of ED patients. Increasing ED capacity has been advocated to increase ED throughput and reduce ED crowding. ${ }^{18}$ Current data on the ability of ED expansion to reduce ED crowding, however, are conflicting. ${ }^{19,20}$

Our overall goal was to determine the effects of ED expansion on measures of ED crowding in our ED. We hypothesized that ED expansion would be associated with a decrease in the rate of patients who left without being treated (LWBT) from the pre-expansion to postexpansion period.

\section{METHODS}

Study Design

This was a retrospective, before-and-after cohort study using data from the University of California Davis health system's electronic health record (EHR) and administrative databases. This study was granted waiver of consent by our institutional review board.

\section{Study Setting and Population}

We performed this study at UC Davis, a single urban, academic ED with an annual volume of approximately 50,000 adult patients. Our hospital is a tertiary care facility with 619 licensed acute care beds and serves a 65,000-square mile area that includes 33 counties and six million residents. The pre-expansion period comprised November 1, 2009, through September 30, 2010. The post-expansion period comprised November 1, 2010, through September 30, 2011. All clinical operations were moved to the expanded ED at 7 am on October 6, 2010. October 2010 was excluded from the study to eliminate the effects that physician and staff adjustment to the new environment may have had on ED operations. No other changes in hospital processes for ED patient flow were made. We included all adult patients (age 18 years and older) who were triaged during the study period.

The old and expanded EDs used a similar operational model, with an increase in adult beds from 33 to 53. The old adult ED comprised two primary treatment "pods" with 12 licensed 
beds each, a three-bed resuscitation area, and a six-bed low-acuity treatment area. The expanded adult ED also comprises two primary treatment "pods" with 12 licensed beds each, a four-bed resuscitation area, and a six-bed low-acuity treatment area. The expanded adult ED also contains two additional treatment pods, one with 12 licensed beds and the other with seven licensed beds.

No substantial changes to the resident physician staffing, nurse-to-patient ratios, or technician-to-patient ratios occurred during 2009 through 2011. State-mandated nursing ratios for the ED and hospital were set at four patients:one nurse for non-intensive care unit (ICU) patients, and two patients:one nurse for ICU-level patients throughout the study period. Starting September 16, 2010, ten hours of attending emergency physician coverage replaced ten hours of physician extender coverage in the low-acuity area of the ED, where patients with Canadian Triage and Acuity Scale ${ }^{21}$ levels IV-V were treated from 9 am to midnight daily. Prior to this time, these patients were treated primarily by physician extenders. Beginning July 1, 2011, physician extenders were fully replaced by attending EPs who provided 15 hours of coverage daily from 9 am to midnight in the low-acuity area.

\section{Study Protocol}

The ED utilizes an electronic health record (EHR) that captures data on every patient who is triaged into the ED system. The following elements from the EHR were downloaded for each day during the study periods: 1) total number of adult patients triaged in the ED, 2) number of adult patients triaged to each of the Canadian Triage and Acuity Scale levels I-V, 3) number of adult patients who LWBT, 4) total wait time in hours for adult patients, 5) total ED length of stay in hours for all adult patients, 6) number of adult patients treated in the low-acuity area, 7) number of adult patients admitted from the ED to the hospital, 8) number of adult patients admitted from the ED to an ICU, 9) total boarding hours for all adult patients admitted from the ED, and 10) number of coded adult trauma activation patients.

The following data were obtained from these administrative records for each day during the study periods: ED nurse staffing hours, number of telemetry/ward beds occupied at midnight, number of ICU beds occupied at midnight, number of elective surgical admissions, number of operational telemetry/ward beds, and number of operational ICU beds.

Wait time was defined as the time from triage to placement in an ED treatment bed. ED length of stay LOS was defined as the time from triage to leaving the ED, regardless of disposition, for patients who were treated in the ED. Boarding hours for admitted patients were included in LOS. The trauma criteria for Level 1, 2, and 3 coded trauma activations are shown in the Data Supplement. Nurse staffing hours include the total number of nursing hours available during the 24-hour period from 7 am to 7 am. Hospital occupancy rate was defined as the percentage of operational hospital beds occupied beds at midnight, and ICU occupancy rate was defined as the percentage of operational ICU beds occupied at midnight.

\section{Outcomes}

The primary outcome measure was the rate of ED patients who LWBT, and the secondary outcome was total daily adult boarding hours. A patient was considered to have LWBT if 
she or he left from the ED waiting room or left from the ED treatment room prior to being evaluated by a physician or physician extender. ED boarding was defined as the interval from one hour following admission bed request placement to the patient leaving the ED to the inpatient bed or operating suite. Boarding hours were calculated based on the patient's date of ED presentation; for example, hours that a patient stayed in the ED beyond midnight on the presenting date would be reflected in the total hours for the presenting date.

\section{Data Analysis}

Summary statistics were calculated for each variable. A multivariable robust linear regression model was used to determine whether ED expansion was associated with changes in the LWBT rate or ED boarding. Robust linear regression was used because some of the outcome data were outliers. Candidate predictor variables were selected based on literature review. ${ }^{17,18,22,23}$ Those considered in the LWBT model included ED adult volume, ED lowacuity area volume, number of coded adult trauma activation patients, total ED wait time, total ED boarding hours, and ED nurse staffing hours. Candidate variables for the ED boarding model included number of ED admissions, number of ED admissions to the ICU, number of elective surgical admissions, hospital occupancy rate, ICU occupancy rate, and number of operational ICU beds. The pre- versus post-expansion variable was forced into the multivariable robust linear regression models. Additional variables were selected into the models using the stepwise method with the significance level of 0.15 for entry into the model, and the significance level of 0.15 for remaining in the model. We used a p-value of 0.05 as the cutpoint for statistical significance. Leverage-point diagnostics were performed using the canonical robust distance, and residuals based on robust regression estimates were used to detect vertical outliers, which are implemented in SAS PROC ROBUSTREG. Residuals were analyzed using kernel density plots and Q-Q plots. Models were reported only when regression diagnostics were met. All analyses were performed using STATA 12.0 (StataCorp LP, College Station, TX) and SAS Version 9.2 (SAS Institute, Cary, NC).

\section{RESULTS}

In the 11 month period prior to ED expansion, 42,896 adult patients were triaged (mean \pm standard deviation [SD] $128 \pm 14$ patients/day; median 128, interquartile range [IQR] 118 to 137 patients/day]. In the 11 month period after ED expansion, 48,358 adult patients were triaged (mean 145, $\mathrm{SD} \pm 17$ patients/day; median 144, IQR 134 to 156 patients/day). A steady increase in patient volume during the post-expansion period was noted (Figure 1). Patient acuity and trauma volume were similar in both periods. The "up front care" area of the ED handled more visits in the post-expansion period. ED nurse staffing increased from a mean of 427 hours per day pre-expansion to a mean of 487 hours per day post-expansion (Table 1).

The LWBT rate remained unchanged, at $9.0 \%(95 \% \mathrm{CI}=8.4 \%$ to $9.5 \%)$ pre-expansion and $8.3 \%(95 \% \mathrm{CI}=7.8 \%$ to $8.9 \%$ ) post-expansion (difference $0.6 \%, 95 \% \mathrm{CI}=-0.16 \%$ to $1.4 \%$ ); however, ED boarding hours increased from 160 hours/day (95\% CI $=152$ to 168 hours/day) to 180 hours/day (95\% CI $=170$ to 189 hours/day). The LWBT rate followed a 
similar temporal pattern over the two periods (Figure 2a), but ED boarding increased steadily in the post-expansion period (Figure 2b).

The change in the LWBT rate from the pre-expansion to post-expansion period was not significant in the multivariable robust linear regression model after adjustment for ED volume, ED low-acuity area volume, ED wait time, ED boarding hours, and ED nursing hours (Table 2). The increase in ED boarding hours from the pre-expansion to postexpansion period remained significant after adjustment for ED admissions, ED ICU admissions, elective surgical admissions, hospital occupancy rate, ICU occupancy rate, and number of operational ICU beds (Table 3).

\section{DISCUSSION}

We found that ED expansion was associated with no significant change in ED throughput as measured by the LWBT rate. We experienced a substantial increase in ED input during the post-expansion period, and we noted a decline in ED output, evidenced by increased ED boarding.

Emergency department input (volume) increased by 13\% from the pre-expansion to postexpansion period, and steadily increased during the post-expansion period. This rate is higher than the national average, ${ }^{3}$ and higher than our institution's prior annual increases, which were below $2 \%$ in the two years prior to the expansion. This "build it and they will come" phenomena is similar to the increased volume seen following an ED expansion project at a different site. ${ }^{19}$ Following the trend in ED volume, we also experienced an increase in ED admissions by three patients per day. With a mean hospital LOS of 4.8 days, ${ }^{24}$ this increase in ED admissions translates to 5,256 more inpatient days per year for the hospital. Future expansion projects should consider and account for the likelihood of higher-than-expected increases in ED volume and admissions following expansion.

The LWBT rate declined initially in the post-expansion period, but once a new equilibrium was established in the expanded ED, ED wait times (data not shown) and the LWBT rate returned to pre-expansion levels. Extrapolating over an entire year, a sustained $1 \%$ decrease in the LWBT rate at the study institution would result in more than 500 additional adult patients being treated. As over $10 \%$ of patients who leave without being treated return to an ED for care within 72 hours, and approximately $4 \%$ are ultimately admitted to a hospital, ${ }^{22,23,25}$ a sustained decrease in the LWBT rate would result in more timely provision of definitive care for patients.

We believe that our ED expansion was not associated with a sustained decrease in crowding for several reasons. First, ED staffing was not substantially increased. In contrast to Miro et al., whose reorganization efforts included a $34 \%$ overall increase in staffing, ${ }^{20}$ our expansion was accompanied by only a $14 \%$ increase in nurse staffing and no substantial changes in physician, technician, or administrative staffing. Second, inpatient treatment areas were not expanded. Thus, the additional ED treatment beds functionally became a boarding area for admitted patients rather than an active treatment area for ED patients. Although the additional boarding hours consumed nursing and ancillary resources, less 
pressure to move these patients to inpatient areas was felt in the expanded ED. In a study similar to ours, Han et al. found that ED expansion was associated with no change in ambulance diversion, and increases in ED LOS and ED boarding. ${ }^{19}$ We identified the same phenomena at our institution, suggesting that these changes are not institution-specific, and that hospitals planning ED expansion should plan to prevent an associated increase in ED boarding hours.

\section{LIMITATIONS}

The retrospective, before-after design of our study is subject to temporal trends and other institutional changes. We selected matched 11-month periods to minimize the effects of seasonal variation in ED volume, illness patterns, and resident training, and we controlled for several possible institutional changes in our multivariable regression model. Individual medical records were not abstracted, but administrative EHR information was downloaded. Thus, potential for abstractor error was minimized. Our findings represent the experience at a single urban academic medical center and may not be applicable to other settings.

While we expanded our ED by 20 beds, we were sometimes unable to use a portion of these beds due to limited nurse staffing and state-mandated nurse-to-patient ratios. Thus, we included nursing hours and ICU admissions to control for these instances. We were not able to evaluate a change in ICU boarding as our institution does not track data on the number of telemetry and ward, vs. ICU boarding hours. Our hospital and ICU occupancy rates were calculated at midnight, and may not reflect mean occupancy throughout the 24-hour day. Given that various measures of ED crowding exist without consensus on a single preferred approach, we selected outcomes that are accepted manifestations of crowding, ,26-28 and that measure different aspects of ED crowding. ${ }^{29}$ While ambulance diversion has been previously used as a global measure of ED crowding, ${ }^{19}$ our hospital policy prohibited ambulance diversion throughout the study period.

\section{CONCLUSIONS}

An increase in ED bed capacity was associated with no significant change in the percentage of patients who left without being treated, and an increase in ED boarding hours. ED expansion alone does not appear to be an adequate solution to ED crowding.

\section{Supplementary Material}

Refer to Web version on PubMed Central for supplementary material.

\section{Acknowledgments}

Grant Support: The project described was supported by the National Center for Advancing Translational Sciences (NCATS), National Institutes of Health (NIH), through grant \#UL1 TR000002. The study's first author was supported by the National Heart, Lung, and Blood (NHLBI) Research Career Development Programs in Emergency Medicine through grant \#5K12HL108964-03.

Beth Morris, MPH 


\section{References}

1. Pitts SR, Pines JM, Handrigan MT, Kellermann AL. National trends in emergency department occupancy, 2001 to 2008: effect of inpatient admissions versus emergency department practice intensity. Ann Emerg Med. 2012; 60:679-86. [PubMed: 22727201]

2. American College of Emergency Physicians. Crowding. Ann Emerg Med. 2006; 47:585.

3. Institue of Medicine. Hospital-Based Emergency Care: At the Breaking Point. Washington, DC: National Academies Press; 2006.

4. Schull MJ, Morrison LJ, Vermeulen M, Redelmeier DA. Emergency department overcrowding and ambulance transport delays for patients with chest pain. CMAJ. 2003; 168:277-83. [PubMed: 12566332]

5. Schull MJ, Vermeulen M, Slaughter G, Morrison L, Daly P. Emergency department crowding and thrombolysis delays in acute myocardial infarction. Ann Emerg Med. 2004; 44:577-85. [PubMed: 15573032]

6. Pines JM, Localio AR, Hollander JE, et al. The impact of emergency department crowding measures on time to antibiotics for patients with community-acquired pneumonia. Ann Emerg Med. 2007; 50:510-6. [PubMed: 17913298]

7. Fee C, Weber EJ, Maak CA, Bacchetti P. Effect of emergency department crowding on time to antibiotics in patients admitted with community-acquired pneumonia. Ann Emerg Med. 2007; 50:501-9. [PubMed: 17913300]

8. Mills AM, Shofer FS, Chen EH, Hollander JE, Pines JM. The association between emergency department crowding and analgesia administration in acute abdominal pain patients. Acad Emerg Med. 2009; 16:603-8. [PubMed: 19549018]

9. Pines JM, Shofer FS, Isserman JA, Abbuhl SB, Mills AM. The effect of emergency department crowding on analgesia in patients with back pain in two hospitals. Acad Emerg Med. 2010; 17:27683. [PubMed: 20370760]

10. Hwang U, Richardson LD, Sonuyi TO, Morrison RS. The effect of emergency department crowding on the management of pain in older adults with hip fracture. J Am Geriatr Soc. 2006; 54:270-5. [PubMed: 16460378]

11. Pines JM, Hollander JE. Emergency department crowding is associated with poor care for patients with severe pain. Ann Emerg Med. 2008; 51:1-5. [PubMed: 17913299]

12. Pines JM, Prabhu A, Hilton JA, Hollander JE, Datner EM. The effect of emergency department crowding on length of stay and medication treatment times in discharged patients with acute asthma. Acad Emerg Med. 2010; 17:834-9. [PubMed: 20670320]

13. Pines JM, Pollack CV Jr, Diercks DB, Chang AM, Shofer FS, Hollander JE. The association between emergency department crowding and adverse cardiovascular outcomes in patients with chest pain. Acad Emerg Med. 2009; 16:617-25. [PubMed: 19549010]

14. Sun BC, Hsia RY, Weiss RE, et al. Effect of emergency department crowding on outcomes of admitted patients. Ann Emerg Med. 2013; 61(6):605-11. [PubMed: 23218508]

15. Richardson DB. Increase in patient mortality at 10 days associated with emergency department overcrowding. Med J Australia. 2006; 184:213-6. [PubMed: 16515430]

16. Singer AJ, Thode HC Jr, Viccellio P, Pines JM. The association between length of emergency department boarding and mortality. Acad Emerg Med. 2011; 18:1324-9. [PubMed: 22168198]

17. Asplin BR, Magid DJ, Rhodes KV, Solberg LI, Lurie N, Camargo CA Jr. A conceptual model of emergency department crowding. Ann Emerg Med. 2003; 42:173-80. [PubMed: 12883504]

18. Derlet RW, Richards JR. Overcrowding in the nation's emergency departments: complex causes and disturbing effects. Ann Emerg Med. 2000; 35:63-8. [PubMed: 10613941]

19. Han JH, Zhou C, France DJ, et al. The effect of emergency department expansion on emergency department overcrowding. Acad Emerg Med. 2007; 14:338-43. [PubMed: 17400996]

20. Miro O, Sanchez M, Espinosa G, Coll-Vinent B, Bragulat E, Milla J. Analysis of patient flow in the emergency department and the effect of an extensive reorganisation. Emerg Med J. 2003; 20:143-8. [PubMed: 12642527] 
21. Beveridge R. CAEP issues. The Canadian Triage and Acuity Scale: a new and critical element in health care reform. Canadian Association of Emergency Physicians. J Emerg Med. 1998; 16:50711. [PubMed: 9610988]

22. Tropea J, Sundararajan V, Gorelik A, Kennedy M, Cameron P, Brand CA. Patients who leave without being seen in emergency departments: an analysis of predictive factors and outcomes. Acad Emerg Med. 2012; 19:439-47. [PubMed: 22506948]

23. Bindman AB, Grumbach K, Keane D, Rauch L, Luce JM. Consequences of queuing for care at a public hospital emergency department. JAMA. 1991; 266:1091-6. [PubMed: 1865541]

24. Centers for Disease Control and Prevention. [Accessed Feb 1, 2014] FastStats. Hospital Utilization (in non-Federal short-stay hospitals). Available at: http://www.cdc.gov/nchs/fastats/hospital.htm

25. Crilly J, Bost N, Thalib L, Timms J, Gleeson H. Patients who present to the emergency department and leave without being seen: prevalence, predictors and outcomes. Eur J Emerg Med. 2013; 20:248-55. [PubMed: 22810850]

26. Schull MJ, Lazier K, Vermeulen M, Mawhinney S, Morrison LJ. Emergency department contributors to ambulance diversion: a quantitative analysis. Ann Emerg Med. 2003; 41:467-76. [PubMed: 12658245]

27. Schneider S, Zwemer F, Doniger A, Dick R, Czapranski T, Davis E. Rochester, New York: a decade of emergency department overcrowding. Acad Emerg Med. 2001; 8:1044-50. [PubMed: 11691666]

28. Forster AJ, Stiell I, Wells G, Lee AJ, van Walraven C. The effect of hospital occupancy on emergency department length of stay and patient disposition. Acad Emerg Med. 2003; 10:127-33. [PubMed: 12574009]

29. Hwang U, McCarthy ML, Aronsky D, et al. Measures of crowding in the emergency department: a systematic review. Acad Emerg Med. 2011; 18:527-38. [PubMed: 21569171] 


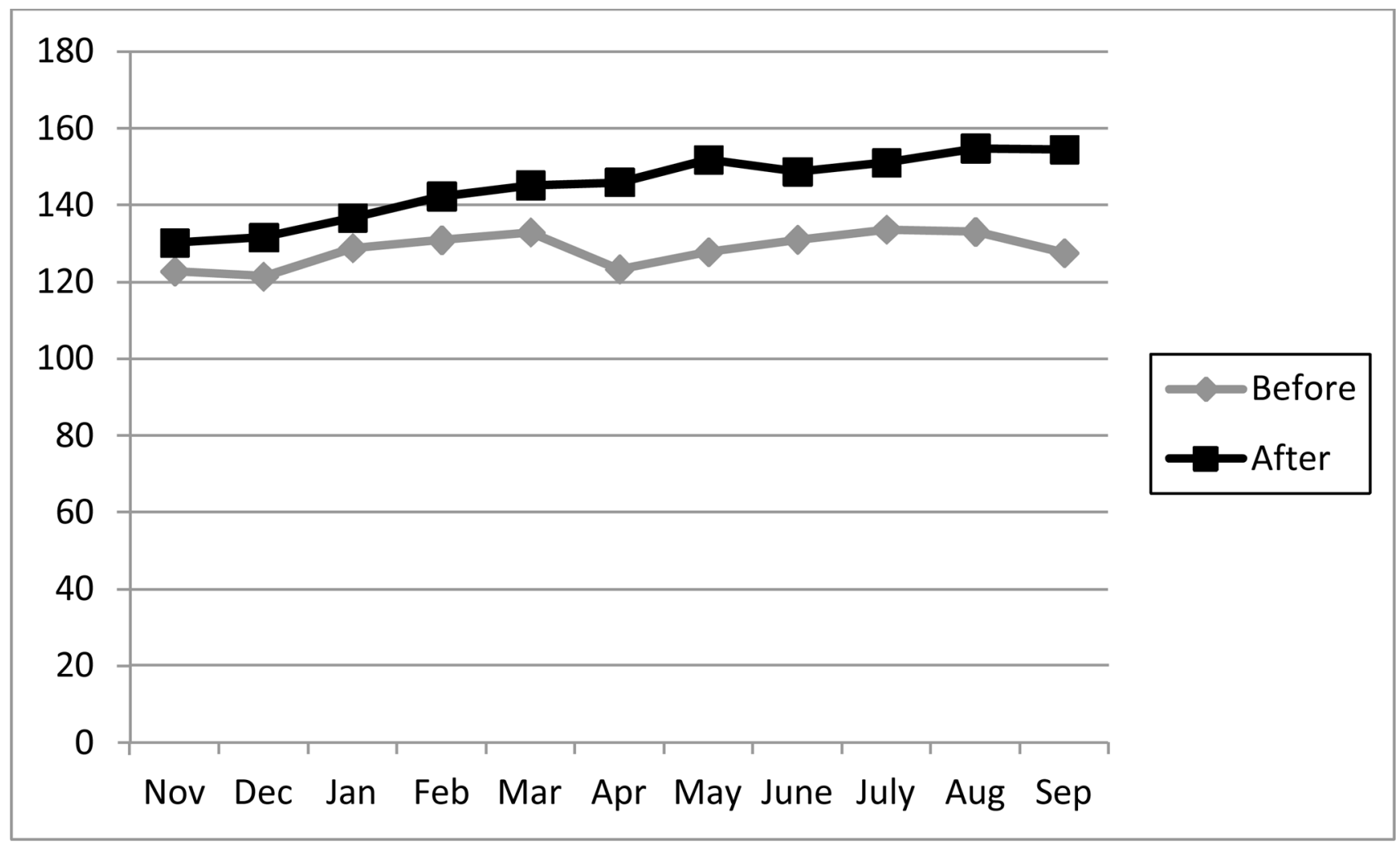

Figure 1.

Daily emergency department volume by month during pre-expansion (before) and postexpansion (after) periods.

Data shown are unadjusted. 
Figure 2a.

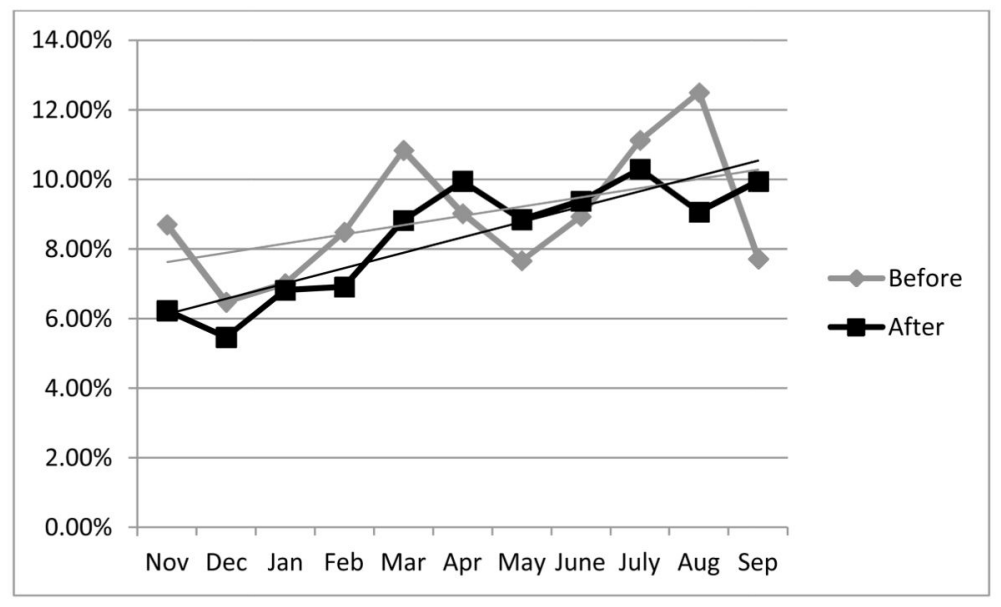

Figure $2 b$.

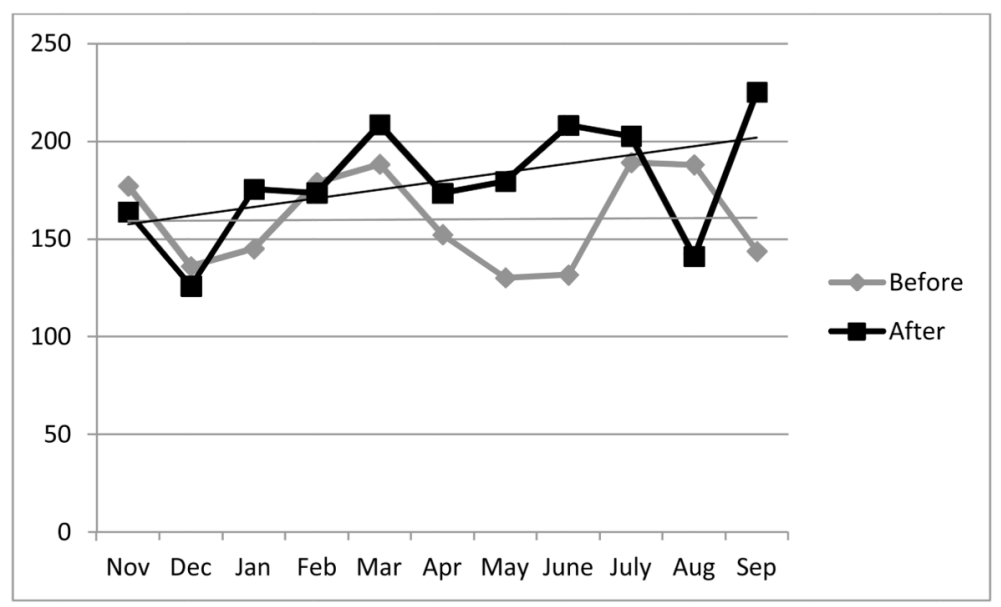

Figure 2.

Figure $2 \mathrm{a}$. Left without being treated rate by month during pre-expansion (before) and postexpansion (after) periods.

Data shown are unadjusted.

Figure 2b. Emergency department boarding hours by month during pre-expansion (before) and post-expansion (after) periods.

Data shown are unadjusted. 


\section{Table 1}

Characteristics of pre- and post-expansion periods.

\begin{tabular}{|c|c|c|}
\hline Characteristic & Pre-expansion $n=42,896$ & Post-expansion $n=48,358$ \\
\hline Daily ED volume & $128 \pm 14$ & $145 \pm 17$ \\
\hline \multicolumn{3}{|l|}{ Triage level, $\%$} \\
\hline CTAS I & 23 & 21 \\
\hline CTAS II & 27 & 28 \\
\hline CTAS III & 38 & 37 \\
\hline CTAS IV & 13 & 14 \\
\hline CTAS V & 0 & 1 \\
\hline Missing & 1 & 1 \\
\hline \multicolumn{3}{|l|}{ Low-acuity area volume } \\
\hline Visits per day & $27 \pm 5$ & $34 \pm 6$ \\
\hline$\%$ of overall volume & 21.0 & 23.2 \\
\hline \multicolumn{3}{|l|}{ Trauma volume (patients/day) } \\
\hline Level 1 & $1.7 \pm 1.3$ & $1.5 \pm 1.2$ \\
\hline Level 2 & $2.2 \pm 1.6$ & $2.0 \pm 1.5$ \\
\hline Level 3 & $6.9 \pm 3.3$ & $6.9 \pm 3.4$ \\
\hline Total adult wait time per day (hours) & $145 \pm 68$ & $148 \pm 77$ \\
\hline Total LOS for all patients (hours) & $934 \pm 184$ & $1027 \pm 218$ \\
\hline Total LOS for discharged patients only (hours) & $556 \pm 120$ & $607 \pm 125$ \\
\hline Total ED admissions per day & $35 \pm 6$ & $38 \pm 6$ \\
\hline Overall admission rate & 27.3 & 26.2 \\
\hline ED admissions to ICU per day & $6.4 \pm 2.5$ & $7.1 \pm 2.5$ \\
\hline ICU admission rate & 5.0 & 4.9 \\
\hline ED nursing hours per day & $427 \pm 23$ & $487 \pm 40$ \\
\hline Elective surgical admissions per day & $13 \pm 9$ & $13.5 \pm 9.2$ \\
\hline Number of hospital beds occupied & $310 \pm 19$ & $316 \pm 20$ \\
\hline Hospital occupancy rate & 79.1 & 80.7 \\
\hline Number of ICU beds occupied & $64 \pm 4$ & $66 \pm 5$ \\
\hline ICU occupancy rate & 97.0 & 93.2 \\
\hline Operational telemetry/ward beds & $392 \pm 0$ & $392 \pm 0$ \\
\hline Operational ICU beds & $66 \pm 0$ & $71 \pm 1$ \\
\hline
\end{tabular}

Data are presented as mean \pm standard deviation or percentage.

CTAS = Canadian Triage and Acuity Score ICU = intensive care unit LOS = length of stay 


\section{Table 2}

Multivariable robust linear regression for the left without being treated rate outcome.

\begin{tabular}{|lcc|}
\hline ED Variable & Parameter Estimate & p-value \\
\hline Expansion & -0.0073 & 0.0532 \\
Volume (pts/day) & 0.0001 & $<0.0001$ \\
Low-acuity area volume (pts/day) & -0.0009 & 0.0001 \\
Wait time (hours) & 0.0004 & $<0.0001$ \\
Boarding hours & 0.0001 & $<0.0001$ \\
Nursing hours & -0.0001 & 0.0944 \\
\hline & & \\
pts = patients & & \\
$\mathrm{R}^{2}=0.5239$ & &
\end{tabular}


Table 3

Multivariable robust linear regression for boarding hours outcome.

\begin{tabular}{|lcc|}
\hline Variable & Parameter Estimate & p-value \\
\hline ED expansion & 43.71 & 0.0049 \\
ED admissions & 4.13 & $<0.0001$ \\
ED ICU admissions & 1.46 & 0.1056 \\
Elective surgical admissions & 1.84 & $<0.0001$ \\
Hospital occupancy rate & 357.71 & $<0.0001$ \\
ICU occupancy rate & 171.03 & $<0.0001$ \\
Operational ICU beds & -9.65 & 0.0016 \\
\hline
\end{tabular}

$\mathrm{R}^{2}=0.3710$

$\mathrm{ICU}=$ intensive care unit 